\title{
Efectividad de un ensayo clínico sobre cuidados enfermeros para la incontinencia en mujeres
}

\author{
Nieto Blanco E, Moriano Bejar P, Serrano Molina L, Dávila Álvarez V, Pérez Llorente M. \\ Unidad de Formación e Investigación. Gerencia de Atención Primaria Área 6. Majadahonda. Madrid.
}

Actas Urol Esp. 2007;31(5):493-501

\section{RESUMEN}

EFECTIVIDAD DE UN ENSAYO CLÍNICO SOBRE CUIDADOS ENFERMEROS PARA LA INCONTINENCIA EN MUJERES

Introducción: La incontinencia urinaria es un problema frecuente en la mujer, se estima que entre el $10 \%$ y el $30 \%$ la padecen Los tipos más comunes son la incontinencia de esfuerzo, de urgencia y mixta. Es un hecho probado que afecta a la calidad de vida.

Objetivo: Evaluar los beneficios derivados de la aplicación de una intervención de cuidados enfermeros, integral y estandarizada en mujeres con incontinencia de esfuerzo, urgencia o mixta, de grado leve o moderada.

Diseño: Ensayo clínico aleatorizado y controlado.

Emplazamiento: Atención Primaria, área 6. Madrid

Participantes: 256 mujeres, 128 por grupo.

Intervención: Plan de cuidados sistematizado para el grupo de intervención y plan convencional para el grupo control.

Resultados: La tasa de participación fue del 59\%, se siguieron 103, 48 en el grupo de intervención (37,5\%) y 55 en el grupo control $(42,9 \%)$.

La diferencia promedio del volumen de pérdidas antes y después de la intervención fue, en las mujeres del grupo de intervención $5,7 \mathrm{~g}(\mathrm{n}=22)$, frente a $1,8 \mathrm{~g}(\mathrm{n}=27)$ en las del grupo control, no siendo esta reducción significativa $(\mathrm{p}=0,12)$, IC al 95\% [$1,09 / 8,92]$. Los cambios producidos en el grado de la IU se valoraron a través de la escala de Sandvik y se agruparon en: "mujeres que se curaron o mejoraron" y "mujeres que permanecieron igual o empeoraron" después de la intervención. Mejoran o se curan el 40,5\% en el grupo de intervención frente al $21 \%$ del grupo control ( $p=0,059)$.

Conclusiones: ambos planes de cuidados se han mostrado eficaces para mejorar la IU de este grupo de mujeres. Ésta ha sido de mayor cuantía en el grupo intervención que en el control, con diferencias clínicamente relevantes. Las pérdidas de mujeres en el seguimiento, condicionó la potencia del estudio.

Palabras claves. Incontinencia urinaria. Mujer. Atención primaria. Enfermería. Ensayo clínico.

\section{ABSTRACT}

EFFICIENCY OF A NURSING CLINICAL TRIAL ON THE TREATMENT OF FEMALE URINARY INCONTINENCE

Introduction: Urinary incontinence is a frequent condition in women; it is estimated that it affects between $10 \%$ and $30 \%$ of women. The most common types are stress, urgency and mixed incontinence. It is a fact that this condition affects their quality of life.

Objective: To assess the benefits of applying an integrated and standardized nursing intervention on women with a slight or moderate degree of stress, urge or mixed urinary incontinence.

Design: Randomized, controlled clinical trial.

Location: Area 6 of primary care in Madrid.

Participants: 256 women, 128 per group.

Intervention: Systematized care plan for the intervention group (IG) and conventional plan for the control group (CG), depending on urinary incontinence type.

Results: $59 \%$ of the total sample participated; 103 participants were followed: 48 from the IG (37,5\%) and 55 from the CG (42,9\%). The average difference in urine loss before and after intervention was $5.7 \mathrm{~g}(\mathrm{n}=22)$ for the IG, as opposed to $1.8 \mathrm{~g}(\mathrm{n}=27)$ for the CG; this reduction was not significant $(\mathrm{p}=0.12), 95 \%$ CI [-1.09-8.92]. IU degree changes were assed by means of the Sandvik scale and were grouped in "women that improved or cured" and "women that remained the same or got worse" after interention. $40.5 \%$ of the women in the IG improved or cured, as opposed to $21 \%$ of the women in the CG (p $=0.059$ ).

Conclusions: both care plans showed their efficiency to improve UI in this group of women. The mentioned improvement was greater in the IG than in the CG, with clinically relevant differences. The fact that some of the women gave up the project during the follow-up determined a power reduction of the study.

Keywords: Urinary incontinence. Women. Primary care. Nursing. Clinical trial.

Este estudio ha sido financiado por el Fondo de Investigaciones Sanitarias del Instituto Carlos III (expediente nº03/671). Responsable de la Unidad de Investigación de enfermería del Área 6 de Atención Primaria de Madrid. 
$\mathrm{L}$ a incontinencia urinaria (IU) es un problema frecuente en las mujeres. Se estima que entre el 10\% y el 30\% la padecen, aunque no sea frecuente que consulten por ello. La incontinencia de esfuerzo (IUE), de urgencia (IUU) y mixta (IUM) son los tipos más comunes en la población general, hallándose prevalencias variables según los instrumentos de medición. Es además conocido que la IU afecta a la calidad de vida (CV), principalmente en las relaciones sociales, siendo las mujeres con IUM las más afectadas ${ }^{1,2}$.

La naturaleza multifactorial de la IU y las circunstancias de cada sujeto aconsejan el diseño de intervenciones que contemplen las distintas particularidades.

El abordaje terapéutico es diverso según el tipo de IU. Así, la rehabilitación del suelo pélvico es el tratamiento conservador de elección para las mujeres con IUE y IUM, tal como evidenció la revisión sistemática de la Cochrane ${ }^{3}$.

En el caso de la incontinencia de urgencia, las recomendaciones bibliográficas sugieren la reeducación del hábito miccional a través de distintos procedimientos que enseñan a controlar la frecuencia y urgencia del deseo miccional.

Conforme aumenta la edad de la población, los costes derivados de la IU se convierten en un factor creciente del gasto sanitario. Moore et al. estudiaron la eficacia y los costes derivados de la terapia para el tratamiento de la IU realizada por enfermeras frente a la terapia uroginecológica, concluyendo que las mejorías encontradas eran parecidas en ambos colectivos a las 12 semanas y a los 2 años, pero los costes eran más bajos en el colectivo de enfermeras. Este autor relata que, recientemente, en los países de la Commonwealth, los profesionales de la enfermería han puesto en marcha programas para el tratamiento convencional de la $\mathrm{IU}^{4}$.

En la revisión bibliográfica efectuada no se encontraron, en el contexto español, ensayos clínicos de esta naturaleza.

La hipótesis de trabajo que se estableció presuponía que una intervención de enfermería estructurada, multimodal y apoyada en la "Teoría del déficit de autocuidados”, resultaría efectiva para mejorar la incontinencia urinaria de esfuerzo, urgencia y mixta de grado leve o moderado en mujeres, frente a la intervención convencional, que no contemplaba un seguimiento estructurado y es la que se ofertaba de forma habitual en la práctica clínica en atención primaria (AP).

Para confirmar esta hipótesis, se realizó este estudio con el objetivo de evaluar los beneficios derivados de la aplicación de una intervención enfermera integral y estandarizada en mujeres con IUE, IUU, y IUM, leve o moderada, desarrollada por enfermeras en el ámbito de la AP.

\section{MÉTODOS}

El diseño del estudio fue un ensayo clínico comunitario controlado y de comparación de una intervención sistemática experimental, frente a la intervención habitual o de control ofertada en la actualidad en los Centros de Salud. La población de estudio fueron mujeres de entre 40 y 69 años de los Centros de Salud del Área 6 de Madrid, estratificada por edad y tipo de IU.

Para evitar la mezcla de sujetos que pudiera producirse entre los dos grupos de intervención, si esta se hubiese realizado dentro del mismo centro de salud, se aleatorizó la muestra por centros en lugar de por individuos. De este modo en cada centro de salud solo se realizó un tipo de intervención.

Se tomó como referencia el $15,4 \%$ de prevalencia que resultó de un estudio epidemiológico realizado en el Área 6 en el año $2000^{2}$.

Siendo conservadores con los resultados de la intervención y considerando que el grupo control de intervención no sistemática obtendría un beneficio limitado (mejoría en el 20\% de las mujeres con IU), se planteó como objetivo de eficacia una mejoría de al menos el 50\% de las mujeres al finalizar la intervención. Para ello se estimó necesario reunir una muestra de 256 mujeres, 128 en el grupo de intervención (GI) y 128 en el grupo control (GC).

La captación de las mujeres se realizó de forma consecutiva, según acudian para ser atendidas en el centro por cualquier motivo, hasta alcanzar el objetivo de la muestra. Las mujeres fueron derivadas de las consultas de enfermería o medicina para su valoración por las enfermeras que participaban en el estudio como investigadoras de campo.

Todas las mujeres que aceptaron participar en el estudio firmaron el consentimiento informado. 
Las variables estudiadas fueron de distinta naturaleza: demográficas (edad, nivel cultural, etc.), clínicas y otras relacionadas con los tipos y grados de IU, con la cantidad de las pérdidas de orina y con el nivel de afectación de la $\mathrm{CV}$. Además se estudiaron aquellas que tenían relación con determinados factores considerados de riesgo para el mantenimiento de la continencia en la mujer, como la amenorrea, el número de partos o la edad. Finalmente se analizaron diversas características de las mujeres consideradas en la literatura científica como potenciales factores desencadenantes de la IU, como son: el consumo de tabaco, fármacos considerados de riesgo (diuréticos, THS, hipnóticos), obesidad o actividad física de riesgo (deportes de alta competición, equitación, aeróbic). De igual modo, se midió la cantidad de líquidos que la mujer tomaba diariamente, el número de veces que se levantaba por la noche y el número de compresas que usaba al día.

También se consideró de interés conocer la opinión de las mujeres sobre si realizaban la micción y la defecación de forma relajada, es decir, sin empujar sobre la vejiga con el diafragma o los abdominales en el momento de la micción, toda vez que este hábito incide en la continencia.

Los criterios de inclusión que se tuvieron en cuenta fueron: mujeres entre 40 y 69 años capacitadas para seguir la intervención propuesta que aceptasen participar en el estudio y con diagnóstico de IUE, IUU o IUM, de grado leve o moderado.

Como criterios de exclusión se consideraron: las mujeres con patología psiquiátrica, enfermedad grave, que tuvieran un prolapso uterino, vaginal o rectal de grado II o III, portadoras de sonda vesical o que tuvieran IU severa o de carácter neurógeno.

Para valorar los tipos de IU se utilizó el cuestionario IU $-4^{5}$, validado y utilizado en nuestro país, que consta de 4 preguntas dicotómicas. El grado de la IU se evaluó mediante la escala de Sandvik ${ }^{6}$, que mide frecuencia y cantidad, clasificándola en leve, moderada y severa de forma subjetiva por la mujer.

Para evaluar el volumen de las pérdidas de orina se utilizó el test de la compresa o pad-test. Las compresas se pesaron con una balanza electrónica (600 g/0,01 g precisión) antes y después de la prueba. Una enfermera ajena al trabajo de campo se encargaba de pesarlas de forma centralizada, antes de las 72 horas.

La afectación que producían los síntomas de la IU en la CV se valoraron con el cuestionario IQOL ${ }^{7}$. Fue autoadministrado y consta de 22 items y 3 subescalas que miden las variaciones de la conducta en 3 esferas: comportamiento de evitación y limitación de la conducta, repercusión psicosocial y sentimiento de vergüenza en la relación social. Las respuestas estaban ordenadas en una escala tipo Likert de 5 puntos, resultando una puntuación entre 0 y 100, correspondiendo la mayor puntuación a la mejor $\mathrm{CV}$.

Tanto a las mujeres del GC como a las del GI se les realizó una primera visita individual, de una hora de duración, cuyo objetivo era la valoración de las variables antes mencionadas y en la que se identificaba el tipo y grado de la IU, lo que permitía establecer el plan de cuidados apropiado.

Para el caso de las mujeres con IUE y IUM del GI, se les propuso un plan de cuidados estructurado y desarrollado en grupo. Este plan constaba de cuatro sesiones educativas semanales y de una hora de duración, con los siguientes contenidos: conocimientos de fisiología y anatomía, descripción y explicación de los factores de riesgo y de los hábitos desencadenantes modificables.

Además se realizaban diversas demostraciones prácticas en el aula sobre ejercicios para el fortalecimiento del suelo pélvico, con las siguientes pautas: contracciones lentas de los músculos de la pelvis manteniendo la tensión 5" y relajándolos 10", se repetía 5 veces seguidas; contracciones rápidas y fuertes de los músculos, relajándolos inmediatamente, también se repetía 5 veces. Esta pauta de ejercicios debía realizarse 10 veces al día en diferentes posiciones: tumbada, sentada o de pie, recomendándose incorporar dichos ejercicios a las actividades de la vida diaria.

A las mujeres del GC con IUE y IUM se les propuso la intervención habitual que realizaban las enfermeras de AP, que consistía en una explicación breve de cómo realizar los ejercicios antes descritos, proporcionándoles una hoja explicativa con la pauta a seguir en su casa.

En el caso de las mujeres con IUU del GI, además de la explicación sobre fisiología y anatomía se valoraba el patrón de eliminación urinaria 
mediante el diario miccional, un instrumento que sirve para conocer los hábitos urinarios: el no $\mathrm{de}$ episodios con pérdida de orina, el tiempo trascurrido entre micciones, el $\mathrm{n}^{\circ}$ de veces que se levanta por la noche y la ingesta de líquidos. Se establecieron los intervalos de eliminación, de acuerdo a su valoración, hasta conseguir evacuaciones cada tres horas.

Además se les enseñaron técnicas específicas para alcanzar un vaciado completo de la vejiga y para poder controlar las situaciones de urgencia (relajación y distracción mental).

A las mujeres con IU del GC se les explicaba individualmente como utilizar el diario miccional según sus hábitos urinarios, así como las pautas a seguir en casa en función de los cambios que fueran consiguiendo.

A las mujeres de ambos grupos que se les detectaron factores de riesgo para la IU modificables se les aplicaron los planes de cuidados específicos en cada caso para mejorar dicho factor de riesgo, como obesidad, tabaquismo, estreñimiento, definido éste como la disminución de la frecuencia normal de la defecación acompañado de defecación difícil o incompleta de heces o eliminación de heces duras y secas.

Se elaboró un manual con los procedimientos a seguir por las enfermeras que realizaron el trabajo de campo, uno para el GI y otro para el GC, especificándose las intervenciones según el tipo de IU, y con el contenido de las sesiones educativas, los ejercicios de rehabilitación del suelo pélvico, las normas para el correcto uso del diario miccional, así como las técnicas de relajación.

La evaluación del plan se realizó a los 3 meses, a partir de la primera visita en el caso de las mujeres del GC, y a los 3 meses de finalizada la intervención grupal en el caso del GI.

Las variables resultado principales que se valoraron fueron: ausencia de pérdidas de orina, ausencia de urgencia miccional, mejora en el grado de la IU, reducción del número de compresas utilizadas, disminución del volumen de las perdidas de orina y mejoría en la $\mathrm{CV}$.

Se analizaron las características basales de las mujeres que abandonaron el estudio en ambos grupos para verificar que no había diferencias estadísticamente significativas entre las mujeres que abandonaron el estudio y las que permanecieron.
En las mujeres que completaron el estudio se realizó un análisis de comparabilidad basal de las variables sociodemográficas, clínicas y sus factores desencadenantes en ambos grupos, mediante los correspondientes test de hipótesis adecuados al tipo y escala de medida de cada variable del estudio $\left(\mathrm{Chi}_{2}\right.$, p exacta de Fisher, $\mathrm{t}$ de Student, U de Mann Whitney) para medias de grupos independientes.

En los contrastes de promedios de valores cuantitativos, se utilizaron los correspondientes test no paramétricos, comprobando previamente la distribución no normal de los valores; si los grupos eran pequeños se aplicó el test de Kruskal-Wallis.

Para conocer la efectividad del plan de cuidados propuesto a experimentación, se valoró si hubo diferencias relevantes entre los cambios apreciados en cada grupo. Así mismo se comparó el grado de la IU, el uso de compresas y la $\mathrm{CV}$ (I-QOl). También se calculó el número necesario de mujeres a tratar (NNT) para conseguir una curación o mejoría de la IU con la intervención propuesta.

Finalmente, para descartar la presencia de fenómenos de confusión significativos en la asociación de la intervención con la mejoría detectada, se utilizó la regresión logística múltiple, ensayando otras covariables potencialmente asociadas a la presencia y al grado de la IU (por el conocimiento bibliográfico previo), o según las observaciones realizadas en el estudio bivariante de asociación.

El análisis estadístico se llevó a cabo mediante el paquete estadístico SPSS versión 10.

\section{RESULTADOS}

De las 256 mujeres que componían la muestra, al $59 \%$ se les hizo la primera valoración basal. El seguimiento se realizó a 103 mujeres: 48 en el GI $(37,5 \%)$ y 55 en el GC $(42,9 \%)$.

Abandonaron el estudio un número similar de mujeres en ambos grupos: 26 en el GI versus 22 en el GC, $\operatorname{chi}_{2}(\mathrm{p}=0,44)$. No se encontraron diferencias estadísticamente significativas entre las características basales de las mujeres que abandonaron y las que permanecieron en el estudio en ninguna de las variables estudiadas: edad, nivel de instrucción, tipo y grado de IU, actividad físi- 
ca de riesgo, IMC, estreñimiento, hábitos miccionales, fármacos, menopausia, $\mathrm{n}^{\circ}$ de partos, $\mathrm{n}^{\circ} \mathrm{de}$ compresas/día, $\mathrm{n}^{\circ}$ de veces que se levanta por la noche, tabaquismo, ingesta de líquidos, café, té, colas, alcohol o si tenían un trabajo considerado de riesgo.

En la Tabla 1 se presentan las características clínicas y demográficas y los factores desencadenantes basales relacionados con la aparición de la IU de las mujeres seguidas. No se encontraron diferencias estadísticamente significativas entre las mujeres del GI y las del GC.

Se analizaron los motivos de abandono expuestos por las mujeres, siendo la falta de tiempo y los problemas de salud personales o familiares los más frecuentes.

La diferencia promedio del volumen de pérdidas antes y después de la intervención fue de 5,7 $\mathrm{g}(\mathrm{n}=22)$ en las mujeres del GI, frente a $1,8 \mathrm{~g}$ en el GC (n=27). Esta reducción no fue estadísticamente significativa $(\mathrm{p}=0,12)$, IC al 95\% [-1,09 8,92] (Fig. 1).

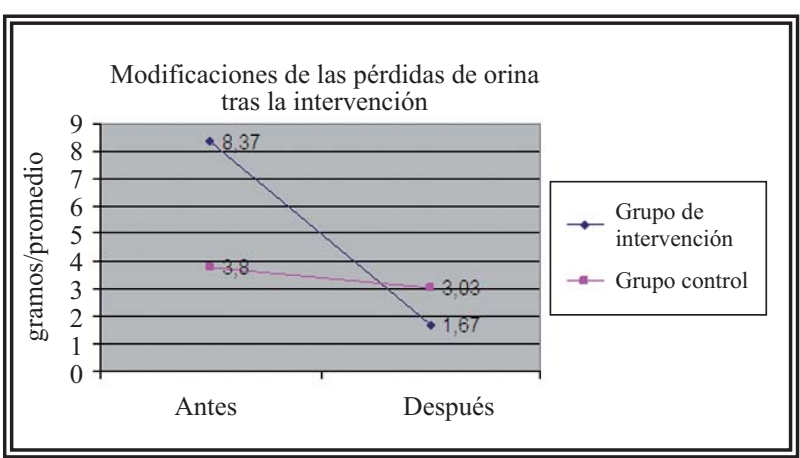

FIGURA 1

Los cambios producidos en el grado de la IU se valoraron a través de la escala de Sandvik, estableciéndose dos categorías para el análisis: "mujeres que se curaron o mejoraron" y "mujeres que permanecieron igual o empeoraron" después de la intervención. Los resultados mostraron que mejoraron o se curaron el 40,5\% en el GI frente al $21 \%$ en el GC, $(\mathrm{p}=0,059)$ (Figs. 2 y 3 ).

El número de mujeres que era necesario tratar con la intervención experimental para conse-

Tabla 1

Comparabilidad basal de los grupos de intervención

\begin{tabular}{|c|c|c|c|}
\hline Variable & Experimental $n=48$ & Control $n=55$ & Valor de $p$ al $95 \%$ \\
\hline Edad: promedio (DS) & $54(8,5)$ & $52(8,3)$ & $* 0,22$ \\
\hline № de compresa (promedio/ día) & $0,92(0,96)$ & $1,03(0,90)$ & $* * 0,79$ \\
\hline Perdida de orina (Pad-test) & $8,3 \mathrm{~g}(\mathrm{n}=37)$ & $3,8 \mathrm{~g}(\mathrm{n}=47)$ & $* 0,06$ \\
\hline IMC (promedio) & $27,4(4,53)$ & $28,4(4,8)$ & $* 0,26$ \\
\hline Total ingesta de líquidos (litros/día) & $1.973(643)$ & $1.832(621)$ & $* 0,27$ \\
\hline № de partos vaginales & $2,2(1,28)$ & $2,1(1,33)$ & $* 0,74$ \\
\hline Vaciamiento completo de la vejiga (si) & $68,8 \%$ & $36,8 \%$ & $* * 0,72$ \\
\hline Control del tiempo entre micciones ( $\%$ no control) & $81,3 \%$ & $72,7 \%$ & $* 0,30$ \\
\hline Toma diuréticos & $30,4 \%$ & $15,1 \%$ & $* 0,06$ \\
\hline Toma tratamiento hormonal sustitutivo & $4,3 \%$ & $9,3 \%$ & $* 0,32$ \\
\hline Se levanta de noche & $68,8 \%$ & $54,5 \%$ & $* 0,14$ \\
\hline Amenorrea & $56,3 \%$ & $58,2 \%$ & \\
\hline \multicolumn{4}{|l|}{ Tipo de IU } \\
\hline IUU & $16,7 \%$ & $10,9 \%$ & \\
\hline IUE & $50 \%$ & $69,1 \%$ & $* 0,14$ \\
\hline IU mixta & $33,3 \%$ & $20 \%$ & \\
\hline \multicolumn{4}{|l|}{ Severidad } \\
\hline Leve & $45,8 \%$ & $50,9 \%$ & $* 0,60$ \\
\hline Moderada & $54,2 \%$ & $49,1 \%$ & \\
\hline Estreñimiento & $29,2 \%$ & $27,3 \%$ & $*_{0} 0,81$ \\
\hline \multirow[t]{2}{*}{ Calidad de Vida (CV) } & - & - & \\
\hline & $\mathrm{x}=76,5$ & $-\mathrm{x}=78,7$ & $* 0,57$ \\
\hline
\end{tabular}

* valor de la p en la prueba U de Mann-Whintney

** valor de la $\mathrm{p}$ en la prueba de $\mathrm{chi}^{2}$ 


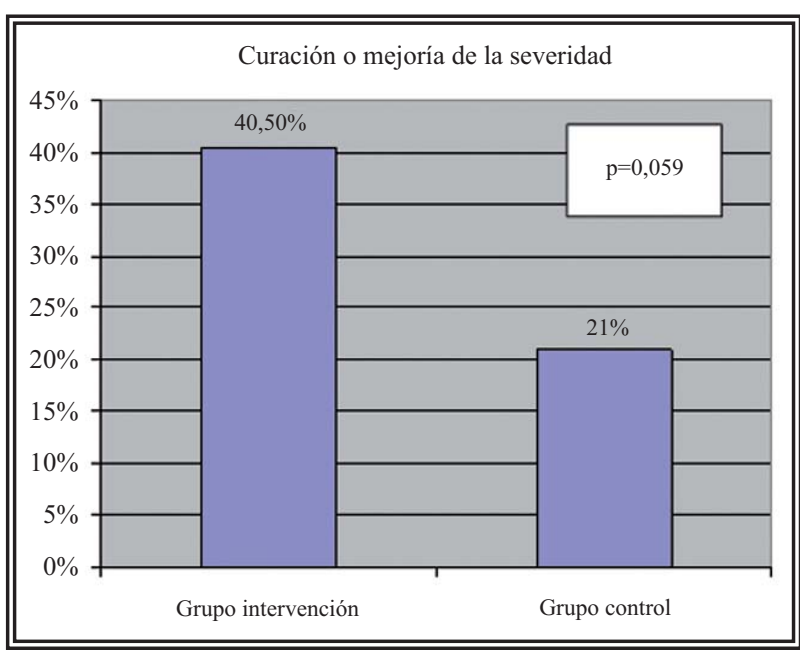

FIGURA 2

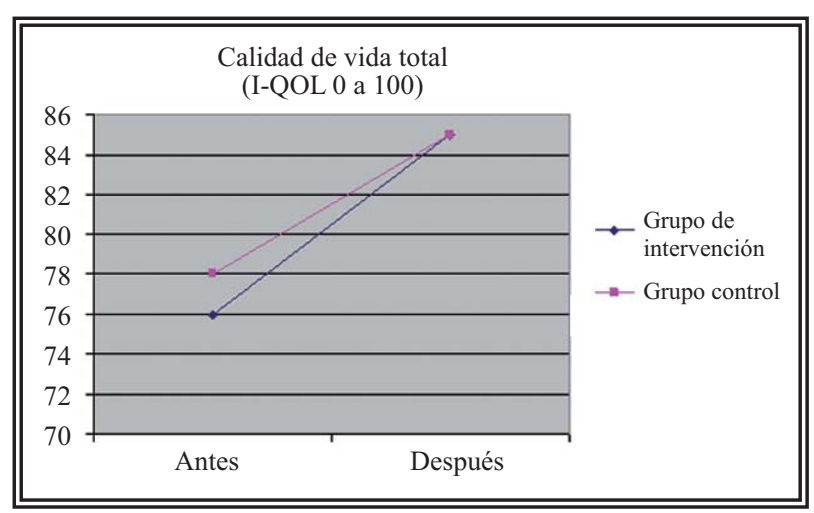

FIGURA 3

guir una "mejoría o curación" fue de 5,2 mujeres, y de 10,5 si se consideraba sólo a aquellas que podrían curarse totalmente.

La utilización de compresas por parte de las mujeres disminuyó en ambos grupos tras la intervención, la proporción de la misma fue de un $60,7 \%$ en el GI versus $36,4 \%$ en el GC, aunque estas diferencias no fueron significativas $(\mathrm{p}=0,16)$.

El cuestionario de CV tras la intervención fue cumplimentado por 37 mujeres $(35,9 \%)$ en el GI y $35(42,7 \%)$ en el GC.

En los resultados de las tres subescalas en que se subdividen los 22 ítems, se apreció que en dos de ellas, "psicosocial" y "relaciones", las puntuaciones mejoraron tras la intervención, tanto en el GI como en el GC. En la subescala de "comportamiento", donde la puntuación disminuyó el GC frente al GI, estas diferencias resultaron estadísticamente significativas $(p=0,000)$.
El promedio de las puntuaciones de la CV tras la intervención se muestra en el gráfico 3. Estas diferencias fueron significativas estadísticamente en ambos grupos: GI ( $\mathrm{p}=0,002)$ y GC ( $\mathrm{p}=0,001)$.

Entre las covariables estudiadas potencialmente asociadas con la presencia y grado de IU no se encontraron asociaciones estadísticamente significativas en ninguna de ellas: el estreñimiento desapareció tras la intervención en un $19,7 \%$ en las mujeres del GC ( $\mathrm{p}=0,014)$, y en un $6,2 \%(\mathrm{p}=0,31)$ en el GI.

La micción relajada en la valoración basal del GI no la realizaban el 10,4\%; tras la intervención, no la realizaban un $4,5 \%$. Estas diferencias no fueron significativas, test Wilcoxon $(p=0,15)$. En el GC, el $18,2 \%$ no la realizaban al inicio, y tras la intervención, todas la realizaban $(p=0,005)$.

El IMC se agrupó en dos categorías: $>31$ y $<31$, calculándose la reducción absoluta del riesgo (RAR). Resultó que el 30\% de las mujeres con IMC menor de 30 tienen una probabilidad de "mejoría o curación" frente al 10,2\% de las mujeres cuyo IMC es superior al 30\%, evaluada esta curación por la desaparición de la IU o reducción de su grado.

\section{Otras variables relacionadas con el efecto}

Se analizó el efecto de la intervención según la edad de las mujeres, que se estratificó en 3 grupos: de 40 a 50 , de 51 a 60 y de 61 a 69 años, observándose que en las más jóvenes la curación o mejoría fue del $50 \%$ en ambos grupos; en el grupo de mediana edad fue del $67,7 \%$ para el GI y 33,3\% para el GC; entre las mujeres del último grupo la mejoría fue del $80 \%$ para el GI y de un $20 \%$ para el GC. Estas diferencias no alcanzaron significación estadística.

De las mujeres que no tenían la regla, "mejoraron" o "se curaron" el $67,7 \%$ y $80 \%$ respectivamente en el GI, frente al 33,3\% y $20 \%$ en el GC. No hubo diferencias estadísticamente significativas entre los grupos.

Considerado el efecto según el tipo de IU, los resultados mostraron que en las mujeres del GI, la "mejoría o curación" se produjo en el 30,7\% de las que tenían IUE, en el 30,7\% con IUM y en el $3,8 \%$ que tenía IUU. En el GC la "mejoría o curación" se produjo en el 11,5\% de las mujeres que tenían IUE, en el 19,2\% de aquellas con IUU y en el $3,8 \%$ cuya IU era mixta. 
Para detectar la posible presencia de fenómenos de confusión en la mejoría apreciada con la intervención experimental se construyó un modelo de regresión logistica multivariante, en el que se empleó la mejoría de la IU como variable resultado y se ensayaron como posibles covariables determinadas características potencialmente asociadas a la incontinencia, según el conocimiento actual (IMC por encima de 30, estreñimiento, actividad física de riesgo, amenorrea, $\mathrm{n}^{\mathrm{o}}$ de partos, histerectomía y cirugía pélvica), y otras que mostraron asociación univariante con la mejoría de la IU (tratamiento hormonal e ingesta de líquidos por encima de 2 litros). Ninguna de ellas ha mostrado una asociación con la mejoría de la IU, ni se modificaron de forma significativa los coeficientes del modelo en el que se incluyó la exploración de la relación de la intervención con la mejoría. Esta asociación se mantiene en el limite de la significación e independiente de la influencia del resto de las variables ensayadas.

\section{DISCUSIÓN}

El presente estudio evaluó la eficacia de un plan estructurado de cuidados enfermeros para recuperar la continencia o mejorar la IUE, IUU o IUM, leve o moderada en mujeres y conducido por enfermeras en el ámbito de la atención primaria.

Las dos intervenciones fueron eficaces en la mejoría de la IU, tanto en el grado como en la reducción del volumen de las pérdidas de orina, en la disminución del número de compresas que previamente usaban las mujeres y en el incremento de las puntuaciones en el cuestionario de CV.

Esta mejoría fue de mayor cuantía en el GI respecto del GC, con diferencias clínicamente relevantes. Sin embargo, los test de hipótesis de comparación del efecto logrado en las distintas variables no han rebasado el límite de la significación estadística $(\mathrm{p}<0,05)$.

Los abandonos de las mujeres durante el seguimiento condicionaron la reducción de la potencia del estudio respecto a la precisión efectuada en los cálculos muestrales (poder de estudio estimado sobre población real seguida: 0,72).

La insuficiencia de la muestra puede justificar la incapacidad del estudio para corroborar esta- dísticamente los hallazgos (error $\beta$ ). En el trabajo de Alewijnse et al. se encontraron problemas semejantes ${ }^{8}$.

La diferencia de la reducción media de las pérdidas logradas con ambas intervenciones fue de 3,9 g. Esta diferencia, interesante sin embargo, hay que tomarla con cautela, ya que puede verse influida por el efecto "suelo" en la medida en que las pérdidas de orina al inicio del estudio fueron menores en el GC que en el GI.

Las mujeres que mejoraron su IU o que recuperaron la continencia son el doble en el GI que en el GC.

La utilización de compresas se redujo en los dos grupos de mujeres; no obstante, esta reducción fue del doble en el GI respecto del GC.

Las mujeres con IUE y IUM mejoraron un tercio más que las que tenían IUU. Resulta dificil conocer las razones de esta diferencia, si bien puede pensarse que la intervención dirigida a las de mujeres con IUU era más compleja, no sólo porque quizás fuera necesario más tiempo para el aprendizaje, sino también porque pudiera ocurrir que las mujeres encontrarán más dificil ver la relación entre la intervención educativa (relajación, distracción mental, diario miccional) y la repercusión que estas técnicas pudieran tener en su mejoría; esto explicaría, en parte, una peor adherencia al plan de cuidados en este grupo de mujeres con IUU.

Los resultados encontrados en relación con la obesidad son semejantes a los de otros estudios y apoyan la multifactorialidad de la IU. En el ensayo clínico de Subak publicado en 2005, se constató que las mujeres que habían adelgazado experimentaron una reducción en el número de pérdidas semanales ${ }^{9}$. Al ser la obesidad uno de los factores asociados, es necesario tenerla en consideración en los planes de cuidados específicos relacionados con el tratamiento de la incontinencia en mujeres.

Los resultados del análisis bivariante pusieron de manifiesto que las mejorías apreciadas en las covariables estudiadas no influyeron en la mejoría detectada en las mujeres.

Las puntuaciones del test sobre la repercusión de los síntomas de la IU en la CV de las mujeres, cuyos valores medios fueron 64,9 y 57,6 respectivamente, fueron altas si se comparan con las puntuaciones obtenidas en otros estudios ${ }^{10,11}$. 
Esta escasa repercusión de la IU en la CV parece acorde con el grado de IU que presentaban las mujeres de este estudio, que era leve o moderado.

Sin embargo, hay que resaltar que las puntuaciones aumentaron en ambos grupos tras la intervención, lo que sugiere que sólo la información y conocimiento del problema que proporcionaban ambos planes de cuidados mejoraban la percepción previa que las mujeres tenían sobre su CV.

$\mathrm{El}$ análisis de las tres subescalas que conforman los distintos ítems del test de CV reveló que, en la escala que se denomina "comportamiento de evitación y limitación”, las puntuaciones empeoraron en las mujeres del GC. Los elementos que componen esta subescala están relacionados con determinadas preocupaciones que les produce la IU y con la planificación de algunas actividades cotidianas que debe tener en cuenta la mujer en función de su IU.

Así mismo se vio que el ritmo del sueño estaba alterado como consecuencia de la incontinencia. En el estudio de Cony SK. de 2004 sobre el impacto de la IU de urgencia en la CV de las mujeres, se encontró que éstas tenían una peor calidad del sueño ${ }^{11}$. Las mujeres con IUM fueron las que tenían peor CV.

Es relevante clínicamente que por cada 5 mujeres que se trataran se conseguiría una mejoría y que para obtener una curación sería necesario tratar a 10 mujeres. Estos hallazgos ponen de manifiesto la efectividad del plan propuesto para ser desarrollado por enfermeros en el ámbito de la atención primaria.

Una cuarta parte de las mujeres abandonaron el estudio en ambos grupos; sin embargo, hay que señalar que la asignación aleatoria de los tratamientos por centro y la homogeneidad de los sujetos que continuaron ha minimizado los posibles sesgos de selección, toda vez que en los análisis realizados no se encontraron diferencias estadísticamente significativas entre las mujeres que permanecieron en el estudio y las que lo abandonaron.

La posibilidad de que se produjeran abandonos fue una de las limitaciones que se señalaron en el proyecto del estudio. El plan propuesto a examen conlleva citas repetidas en el centro, en horarios no siempre acordes a las necesidades de las mujeres. Son las más jóvenes las que más abandonaron. En parte, cabe suponer que muchas trabajan fuera de casa, lo que dificultó las citas, aunque este aspecto no se midió.

Todas estas circunstancias, aunque se tuvieron en cuenta, no pudieron solventarse en su totalidad, a pesar de que se utilizaron distintos procedimientos para recaptar a las mujeres.

Por otro lado, los autores piensan que el test de la compresa contribuyó a los abandonos, ya que fue la variable con la tasa de respuesta más baja. Tal hecho sugiere que el tiempo que consumía este procedimiento fue el motivo primordial de la baja tasa de respuesta en esta prueba, la cual requería personarse en el centro varias veces. Existe alguna controversia en la literatura acerca de la fiabilidad de la prueba, si bien es uno de los indicadores objetivos para medir el efecto y fácil de utilizar en el ámbito de la atención primaria.

Existen algunos puntos débiles en este trabajo y uno de ellos es el efecto Hawthorne; todos los enfermeros participantes conocían la hipótesis del estudio y, dado que en este tipo de diseños resulta difícil enmascarar la intervención, pudiera suceder que los enfermeros correspondientes al GI pusieran mayor entusiasmo en el proceso.

Al contabilizarse como pérdida de orina volúmenes muy bajos (cualquier valor por encima de $0 \mathrm{~g}$ ), pudo suceder que en las mujeres con pérdidas muy pequeñas no se produjeran reducciones apreciables, lo que induciría a pensar que la fiabilidad de la prueba en estos casos no es la adecuada. Turkan ${ }^{12}$ considera que por debajo de $1 \mathrm{~g}$ no hay incontinencia, ya que pérdidas entre 0 y 1 $g$ pueden deberse a fluidos vaginales o al sudor. Ésta es una cuestión a valorar en futuros trabajos.

Como ya ha sido comentado, la población del estudio presentaba una IU en grado moderado o leve, características éstas más fáciles de detectar en el ámbito de la atención primaria que en los servicios de atención especializada a los que acuden generalmente las mujeres con IU severa. Los resultados que se exponen son representativos de este tipo de mujeres.

Se precisarian proyectos específicos para replicar el estudio en otros ámbitos de atención y con mujeres cuya IU fuera más grave. 
Es importante realizar el diagnóstico e iniciar el tratamiento en las fases precoces de la IU, acciones más sencillas y propias de ser realizadas por los profesionales de la atención primaria.

Finalmente, nos gustaría señalar que estudios de esta naturaleza, en los que se pretende analizar la efectividad de una intervención enfermera en el ámbito de la atención primaria y desde la práctica clínica, son un reto en nuestro país y precisarían ayudas específicas para el trabajo de campo.

La intervención propuesta mejoró la IU en mujeres de mediana edad, al reducir el volumen de las pérdidas, mejorar el grado de su IU, disminuir el uso de material protector y aumentar los valores asociados a la $\mathrm{CV}$, aunque los abandonos habidos limitan la potencia del estudio.

Los resultados son clínicamente relevantes, sobre todo en mujeres que tenían IUE y IUM, como evidenciaron los resultados de los NNT.

La enfermera comunitaria es uno de los profesionales sanitarios competentes para llevar a cabo estos planes de cuidados en su práctica clínica.

Es necesario seguir investigando en nuestro contexto sobre las lagunas e incógnitas que aún quedan sobre los cuidados de la IU y más concretamente en el caso de la IU de urgencia.

\section{Agradecimientos}

A todo el grupo de enfermeros que participaron en el trabajo de campo, sin cuya dedicación no habría podido realizarse este trabajo.

A Fernando Caballero Martín por su inestimable ayuda metodológica.

\section{REFERENCIAS}

1. Damián J, Martín-Moreno JM, Lobo F, Bonache J, Cerviño J, Redondo-Márquez L et al. Prevalence of urinary incontinence among Spanish older people living at home. Eur Urol 1998;34(4):333-338.

2. Nieto Blanco E, Camacho Pérez J, Dávila Álvarez V, Ledo García P, Moriano Bejar P, Pérez Llorente $\mathrm{M}$ et al. Epidemiología e impacto de la incontinencia urinaria en mujeres de 40 a 65 años de edad en un área sanitaria de Madrid. Aten Primaria 2003; 32(7):410-414.
3. Hay-Smith, E.J.C, BØ K, Berghmans, L.C.M, Hendriks, et al. Pelvic floor muscle training for uninary incontinence in women (Cochrane Review).In: The Cochrane Library Oxford: Update Software, 2003.

4. Moore K.H, O’Sullivan RJ,Simons A, Prashar S, Anderson M, Louey M. Randomised controlled trial of nurse continence advisor therapy compared with standard urogynaecology regimen for conservative incontinence treatment: efficacy, costs and two year follow up. BJOG.2003 July; 110(7):649-657.

5. Badia X, Castro D, Perales L, Pena JM, Martínez-Agulló E, Conejero $\mathrm{J}$ et al. Elaboración y validación preliminar del cuestionario para la clasificación clínica de la incontinencia urinaria. Actas Urol Esp. 1999,23(7):565-572.

6. Sandvik H, Steinar H, Arnfinn S, Hermstad R, Vanvik A, Bratt $H$. Validation of a severity index in female urinary incontinence and its impletation in an epidemiological survey. J Epidemiol Community Health. 1993 Dec;47(6): 497-499.

7. Patrick DL, Martin ML, Bushnell DM, Yalcin I, Wagner TH, Buesching DP. Quality of life of women with urinary incontinence: further development of the incontinence quality of life instrument (I-QOL). Urology 1999 Jan;53(1):71-76.

8. Alewijnse D, Metsemakers JF, Mesters IE, van den Borne BH. Effectiveness of pelvic floor muscle exercise therapy supplemented with a health education program to promote long-term adherence among women with urinary incontinence. Neurourol Urodyn 2003;22(4):284-295.

9. Subak LL, Whitcomb E, Dhen H, Inttinghff E, Brown JS. Weight loss: a novel and effective treatment for urinary incontinence. J Urol. 2005 Jul; 174(1): 190-195.

10. Cony KS, Payne C, Bhattacharyya SK, Revicki DA, Thompson C, Corey R et al. The impact of urinary urgency and frequency on health-related quality of life in overactive bladder: results from a national community survey. Value Health.2004 Jul-Aug; 7(4):455-463.

11. Nieto Blanco E, Camacho Pérez J, Dávila Álvarez V, Ledo García P, Moriano Bejar P, Pérez Lorente M et al. Repercusión de la incontinencia urinaria en localidad de vida de las mujeres de 40 a 65 años en un área sanitaria de Madrid. Enfermería Clínica 2004;14(3):129-135.

12. Turka A, Inci Y, Fazli D. The short-term effects of physical therapy in different intensities of urodynamic stress incontinence. Gynecol obstet invest. 2005;59(1):43-48.

Correspondencia autora: Dña. Esther Nieto Blanco Unidad de Formación e Investigación de Atención Primaria Área 6 Avda. España, 7 - Majadahonda. Madrid.

Tel.: 916026944

E-mail autora: enieto.gapm06@salud.madrid.org

Información artículo: Original - Urol. funcional - Incont. femenina Trabajo recibido: febrero 2007

Trabajo aceptado: marzo 2007 